\section{Technigue of Treatment}

As regards the technique of the treatment, it may bie stated that since the sea climate imposes a certain strain upon the body, it must be carefully applied. The individual reaction cannot be known beforehand. With delicate persons it is well to prescribe at first lying down; with nervous children the stay on the beach itself must be limited. With many other forms of sickness, on the contrary, a benefit is only to be obtained through remaining close to the sea, especially with susceptible respiratory organs and retarded development. Care of the skin forms an essential part of our whole treatment. The often astonishingly relaxed skin is rendered tonic and active by dry rubbing, simple washing with cold sea water, air and sun baths, warm sea baths followed by cold shower baths, or by cold sea baths. Here is the place to state expressly that the sea climate is not to be plaved with. We see each year the most incredible follies and extravagances in the treatment of children at the seaside, amounting almost to brutalities of parents towards their children. Such are the prolonged and repeated sea and sun baths, endless wading in the water or unlimited stay on the beach and in the wind. In all our remedial measures very brief, often repeated, stimulations are the most effective, while prolonged cold baths, for instance, have the contrary effect and defeat the hardening process, which is our objective. With school work also one must be careful at the seaside. It is not surprising to me that an English observer, Dr. Marvel, said " he doubted whether sea baths did not produce more harm than good." Russel cautioned, too, against dangerous exaggerations :

Marina aqua . . . . magna vi pollet; sed imperiti facile ipsa perperam uti possunt.

Even Homer knew this action of the sea to be very exacting :

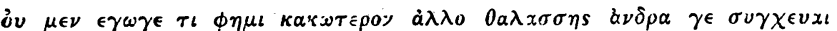

Gymnastics are an important part of our cures. As in the colder season air baths are only possible with quicker motion, games are indispensable.

One has to be very careful about the diet of dclicate children at the seaside. Suitable food powerfully assists in the process of healing, and in children prone to exudations dietetic measures are indispensable. In winter we use a quartz lamp, vitamin preparations, and sometimes arsenic and iodine, and often local treatment for adenoids. At marine hospitals with large numbers of children infections may play a large part, which has led a German paediatrician to use the same words as did Dr. Marvel on account of the danger of overdosing. Quarantine of all newcomers should be considered, and perhaps active immunization-for instance, against diphtheria. Both these measures are practised in France.

It is obvious that a short period of treatment will not suffice for chronic or constitutional diseases such as those which are mostly sent to us. We have to aliow four weeks for acclimatization, which represents a certain allergical stage. Then only the benefit of our climate begins to deveiop fully. In general a period of less than three months' duration would not have the desired and lasting result. In more serious cases of catarrhs, asthma, debility, imperfect development, from half to one year, or even more, is necessary. To interrupt this period by a change of climate is generally inadvisable so long at least as we sce a child deriving benefit. There are naturally some patients with whom one has an impression from the beginning that the sea climate does not agree. These will be sent as soon as possible to another climatic resort. It should be especially noted that for most respiratory catarrhs, and for asthma, treatment is more important in winter than in summer, and should correspond with the winter climax of these affections.*

The increase of weight in the children at Wyk auf Föhr is at its maximum in autumn. We have also noticed that the more serious retardation of development; which in summer shows little progress, is all at once improved in the autumn.

On the whole the sea represents such a powerful source of invigoration obtainable for a great part of mankind that we can fully agree with the saying of Russel almost 200 years ago :

rem de qua agimus nec levem esse, sed dignam principis viri tutelae utpote quae ad universam populi valetudinam consc $r$ vandam spectat.

\section{PRURITUS ANI $\dagger$}

BY

\section{W. P. MACARTHUR, M.D., F.R.C.P.I.}

BREVET-COLONEL, R.A.M.C.; CONSUITING P'HYSICIAN TO THE BRITISH ARNYY

I have been asked to discuss the varicty of pruritus ani of adults caused by Enterobius vermicularis, a nematode now removed from the genus Oxyuris for the reason that its characters do not conform to those of the genotype, Oxyuris equi. To some this contribution will seem un-alled for, but so long as one can encounter persons who for years have endured the nightly torments of enterobiasis without the true cause of the pruritus bing discovered, or its nature even suspected, an apology for dealing with so commonplace a subject is unnecessary.

The ingested ova of Enterobius hatch in the duodenum. The larvae at first inhabit the small intestine, and later migrate to the caecum, where the majority of the worms exist. The appendix is a favoured habitat. About two wecks are required to complete the cycle from ovum to ovum. In 5 instances of infection in which $\mathrm{I}$ obtained strong evidence indicating the source, the symptoms in 4 commenced on the 15th day; and in 1 , on the 14 th, after the apparent exposure. The gravid females make their way to the rectum and anus to oviposit, and if not repelled by the cold they may be seen thus engaged just inside the anal canal. It is their wriggling movements which cause the irritation loosely called pruritus; it is not an itching, however, but a very quick, light, tickling sensation, most intolerable to the victim. Worms may be found outside the intestinal canal in the neighbourhood of the anus, on clothing, etc., but $I$ do not know if this complete emergence is voluntary, or if the parasites have been displaced in scratching. They have considerable powers of resistance to expulsion by natural means, for an ordinary evacuation may leave an active pruritus quite unaffected. Possibly they escape this danger by retiring into the rectum, as they can be observed to do when exposed to cold.

As there is no multiplication of parasites within the body the number infecting the host is determined by the number of ova swallowed. Aito-infection by the mouth is the rule, and appears to be specially provided for by nature. An infestation which persists for more than about six weeks is almost certainly kept up in this way. Although the host reinfects hirnself with the utmost readiness, it is curious that members of his family in daily contact with him for years usually escape contamination.

* A seasonal physiological pericdicity of certain respiratcry functions is known. The alveolar $\mathrm{CO}$, tension is in winter 42.5 , in tions is know. line alveolar co, tension is in winter 42.5, in summer 36 (Lindhard, Straub), the frequency of breathing is also higher in whinges result could not be obtained in England (Haldane, Priestley and others) seems due to the island character of the British climate, which cannot show the same meteorological contrasts as the climate of continents. $\dagger$ Read in opening a discussion in the Section of Dermatology at the Annual Meeting of the British Medical Association, Eastlourne, 1931 . 
Enterobius sets up a catarrh of the intestine, and the accornpanying increased secretion of mucus may be very obvious. The mucous membrane of the lower part of the rectum is congested and usually shows small haemorrhagic spots, a condition which probably extends throughout the intestine, as similar changes have been repeatedly found in the appendix. Some degree of eosinophilia is the rule, and this suggests that the general and nervous symptoms may not entirely be due to sleeplessness and mental distress.

\section{Symptoms}

Pruritus, so called, is the most constant symptom. It may commence very suddenly, and the subject of an infestation of long standing may remember the exact moment when it was first felt. If only a very few parasites are concerned their individual movements may be distinguished one from the other, but with large numbers the sensation is continuous. Even one worm may make itself felt. In two of my cases there was a distinct recurrence of pruritus after treatment had been completed ; in each the capture of a single Enterobius, gave immediate relief without other treatment.

The pruritus is mainly nocturnal, commencing shortly after the patient goes to bed, and quickly reaching its maximum intensity. Especially in cold weather the patient may get to sleep, only to be wakened later. A hot-water bottle in winter hastens the onset. In severe infestations he may be driven almost demented by the irritation, losing all control of himself, and tearing at the affected part until he draws blood. Some discover for themselves that vigorous movements of a finger inserted into the anal canal may give relief, due, as we know, to destruction of some of the worms. The itching ceases sometime before morning, and is never felt, say, about 5 or 6 o'clock, nor before rising. I should consider an absence of this morning remission strong evidence against enterobiasis. Some itching may be felt more or less intermittently during the forenoon and afternoon, especially after exercise. In the evening, irritation is more likely to be troublesome, and is intensified by a warm atmosphere, and by the proximity of a fire; the patient often notices a sudden relief when he commences to undress in a cold room. But the daily irritation, if present, is always slight in comparison with that felt at night. One of my patients used to speak of going to bed as " descending into hell."

The discomfort and loss of sleep thus caused lead to mental irritability and despondency, which are aggravated by the fact that the sufferer often conceals his infirmity through some feeling of delicacy, and so his family do not make allowance for what they regard as mere bad temper. In the end he reaches a pitiable state of depression, and may become tearful when describing the miseries he endures.

Various reflex phenomena may develop, such as gastric hyperacidity, indigestion-probably due in part to intestinal catarrh-and frequency of micturition. The significance of these symptoms may not be appreciated. One patient complained to me of frequency of micturition of a few weeks' duration. Various examinations and tests were carried out before it was discovered that he had suffered from nocturnal pruritus-which proved to be due to threadworms-for over a year. Another case is still more instructive.

The gentleman in question had been afflicted with pruritus for over ten years. Symptoms of gastric hyperacidity and indigestion had persisted more or less throughout this period. These were first attributed to duodenal ulcer and an appropriate course of treatment was prescribed, but without lasting relief. Next chronic appendicitis was diagnosed and the supposed offender removed, but with a similar absence of benefit. Presumably the appendix contained no threadworms. Later, owing to the quantity of mucus passed, dysentery was sug- gested, and emetine administered, followed by a course of stovarsol, but the symptoms were unaffected. Throughout this period many forms of local treatment, including $x$ rays, had been given for the pruritus, which persisted notwithstanding. Ten years after the onset, enterobiasis was suggested for the first time, a diagnostic rectal wash-out showed the presence of many threadworms, and a course of salt and water injections ended the irritation and the whole train of secondary troubles.

The excessive production of intestinal mucus, already mentioned, may result in the involuntary passage of the secretion, especially when the patient takes exercise or drives a car. It may be troublesome enough to necessitate the wearing of a pad of cotton-wool.

Enterobiasis occasionally causes attacks of appendicular colic leading to removal of the appendix, which when examined is found to contain worms. This experience has suggested appendicectomy as a curative measure in persistent infestations, a wholly unjustifiable procedure, for persons who have previously undergone this operation for any reason are just as liable to infestation as others, and are no easier to cure. The activities of the parasites within the appendix, and the resulting damage to the mucous membrane, may lead to the development of acute appendicitis.

Owing to laceration and abrasions of the anal region, an eczematous condition may be set up, which quickly disappears when the parasites are removed. On the other hand, there may be surprisingly little local injury after years of nightly scratching.

The old nursery symptom of nasal itching may be in evidence. Possibly this is a provision of nature for insuring that the infected hands are brought into the neighbourhood of the mouth. The wife of one patient who eventually proved to have enterobiasis told the medical attendant, months before the condition was diagnosed, that she thought her husband must be suffering from " worms" because he was "always scratching his nose"!

\section{Diagnosis}

In every case of nocturnal pruritus ani, enterobiasis should be considered as a possible cause, and the suspicion either confirmed or negatived by further examination. The most thorough diagnostic measure is a rectal wash-out which must be administered when pruritus is active. If a satisfactory search cannot be carried out because of the presence of faecal matter, the rectum should be cleared by an ordinary soap and water enema given in the evening, before pruritus commences. The patient then goes to bed, and a wash-out with normal saline is given later when pruritus becomes troublesome. Three or four ounces of saline is all that is required, and after a few minutes this is returned into a suitable receptacle, and examined for worms. This examination must be made carefully, and if living worms are not seen, suspicious-looking objects should be removed and examined under the low power of a microscope, or with a hand lens. It is surprising how easily shreds of mucus, vegetable matter, etc., may be mistaken for Enterobius if not removed and examined.

Microscopical examination of the faeces for ova is of little help. Occasionally ova may be found, but their absence signifies nothing. A positive result is much more likely if scrapings from the neighbourhood of the anus, or some rectal mucus, are examined.

A very useful preliminary test is for the patient to give himself a wash-out with strong salt and water (sce under Treatment) when the pruritus is at its height. - If this ceases instantly, and does not return until the following night, the diagnosis of enterobiasis is practically certain. 
It is surprising how seldom, in adults, worms are seen in the faeces. One patient of mine, a medical man, looked at every motion passed during a period of several weeks. Although nocturnal pruritus was constantly present, only a single worm was sten, and this in a motion resulting from a sharp purge given as part of his treatment.

\section{TREATMENT}

Prolonged infestation always results from auto-infection by the mouth, and until this source of supply has been cut off no treatment can be effective. The ova are deposited in enormous numbers in and around the anal region. They may reach the mouth either directly by the hands or they may be smeared over the body and clothing, and the hands reinfected later from these sources. Crushing of worms either in scratching or otherwise liberates eggs in large numbers. I have recovered several dozen eggs from a single finger-nail, and Professor D. B. Blacklock tells me that he has found ova in washings made from garments as low down as the knee. From this it is obvious that the washing of the hands before food and after defaecation, as often directed, is totally inadequate. The hands must be washed thoroughly every time that the surface of the body, or an undergarment, is touched, for there is no certainty that eggs have not been picked up. For example, on waking, the hands must be washed before the morning cigarette and cup of tea. Suppose the patient next rises and removes a vest, then another washing is necessary before he shaves, or cleans his teeth. In dressing, further contamination is likely, therefore the hands must be washed again before breakfast, and so on throughout the day. Laxness in this respect will be followed by vexatious reinfections. Even with scrupulous care, occasional disappointments will be experienced. Nature is unceasing in the attempt to complete the life-cycle of the parasites, and if auto-infection is prevented the infestation will die out of itself.

Treatment proper falls under two heads: first, the removal by lavage of the adult female worms which have migrated to the rectum, and second, attempts to destroy the developing stages in the caecum, appendix, and neigh bouring parts of the intestine. For the first-mentioned purpose nothing is superior to salt and water in a strength of 2 tablespoonfuls to the pint. If this should cause griping, reduce the salt by half, and increase later if possible Four ounces of the solution is sufficient for an injection, which can be given most readily by means of an infant's 2-oz. enema bulb, the patient remaining recumbent, with the hips raised, during the operation. Probably he will be unable to retain the solution for more than one or two minutes, and when it is ejected the itching is found to have ceased completely, and is not felt again during the night. It is essential that the patient should administer this treatment for himself, because it is useless if given at any time that pruritus is not felt. A preliminary simple enema, more elaborate apparatus, and larger quantities of fluid, are unnecessary complications. When a sufferer who has been wakened nightly for months or years finds that a simple procedure completed within a very few minutes will protect him from the tortures of pruritus, at once his whole outlook on life is altered.

The second part of treatment is the administration of anthelminthic drugs in the hope of destroying the parasites before they migrate to the lower portions of the large intestine. It is difficult to assess their respective values, for most of the preparations recommended are either given with some laxative or themselves act as purgatives, and a sharp action of bowels due to any cause may give a temporary relief from pruritus; for this reason sufferers from undiagnosed enterobiasis sometimes attribute their troubles to constipation. I do not know of any drug so efficacious as to do away with the necessity for rectal irrigations. Indeed, it is difficult to see how anthelminthics can reach worms lurking in the appendix and caecal pouches, and so long as even two or three are left they may suffice to drive the host into a state of frenzy. On the whole, my experience has been that when a patient finds that a rectal injection gives instant relief from his sufferings, he prefers not to be inconvenienced by purgative drugs, even though his course of treatment might be shortened thereby. Of the preparations usually recommended, I have found 2 grains of santonin combined with $\frac{1}{2}$ a grain of calomel distinctly helpful. Stovarsol had no appreciable effect. Carbon tetrachloride is. reported to be very efincacious, but its employment is hardly justifiable in simple enterobiasis which can be cured with certainty in other ways. Oil of chenopodium and butolan I have not tried.

Treatment, especially lavage, may affect the remarkable periodicity of Enterobius, the commonest change being an earlier descent of the egg-laying females, so that pruritus may be troublesome before bed-time. But whenever it may appear, a rectal irrigation with salt and water will stop it at once. Rectal irrigations, given as advocated, do away altogether with the necessity for application of mercurial ointments and the like.

I do not know to what extent pruritus ani is met with in general practice, nor can I say what proportion of these cases is due to enterobiasis. My submission is that entcro. biasis should be considered as a possible cause in every case of pruritus ani in adults.

\section{'TREA'TMENT OF GENERAL PARALYSIS OF THE INSANE BY INDUCED MALARIA}

\section{NOTE ON FIFTY CASES}

BY

\section{R्OBERT LEES, M.B., CH.B., M.R.C.P.ED.} EDINBCRGH

Since the introduction on a large scale of malarial treatment of general paralysis of the insane by Wagner von Jauregg in 1917, great interest has been aroused in the subject, and this therapentic measure has been extensively practised by numerous workers. The literature is already considerable; it has been fully summarized by G. de M. Rudolf, ' by Surgeon Rear-Admiral Meagher," and by J. Gerstmann. ${ }^{3}$

This communication is intended as a preliminary note on a series of fifty cases which have been treated in Edinburgh under the auspices of the Corporation venereal diseases clinic. The majority of the cases have been treated in the wards of Edinburgh Royal Infirmary, the remainder in Corporation hospitals. This series differs from most others reported in that, without exception, the cases were first seen in the out-patient department of a venereal diseases clinic or in consultation in the medical wards of a general hospital. None of the patients was certified as insane; many of them were at the earliest stage at which the disease may be recognized; only a few_ were at an advanced stage, at which they might have been certified insane. The series includes a relatively high proportion of cases of juvenile general paralysis of the insane (28 per cent.). In practically all the cases under review, treatment by therapeutic malaria has been followed by the administration over a prolonged period of antiluetic drugs.

In treating these patients our aim was to secure lasting remission of the mental symptoms and restoration of physical vigour; as a result of the combined treatment success has been achieved in a gratifying proportion of cases. Accordingly, we have extended the use of this method of treatment since we started our work in 1927. 